\title{
Ground state of nonassociative hydrogen and upper bounds on the magnetic charge of elementary particles
}

\author{
Martin Bojowald, ${ }^{1, *}$ Suddhasattwa Brahma๑, ${ }^{2, \dagger}$ Umut Büyükçam, ${ }^{1, \ddagger}$ and Martijn van Kuppeveld ${ }^{1, \S}$ \\ ${ }^{1}$ Institute for Gravitation and the Cosmos, The Pennsylvania State University, \\ University Park, Pennsylvania 16802, USA \\ ${ }^{2}$ Department of Physics, McGill University, Montréal, Quebec H3A 2T8, Canada
}

(Received 13 August 2021; accepted 19 October 2021; published 10 November 2021)

\begin{abstract}
Formulations of magnetic monopoles in a Hilbert-space formulation of quantum mechanics require Dirac's quantization condition of magnetic charge, which implies a large value that can easily be ruled out for elementary particles by standard atomic spectroscopy. However, an algebraic formulation of nonassociative quantum mechanics is mathematically consistent with fractional magnetic charges of small values. Here, spectral properties in nonassociative quantum mechanics are derived and applied to the ground state of hydrogen with a magnetically charged nucleus. The resulting energy leads to new strong upper bounds for the magnetic charge of various elementary particles that can appear as the nucleus of hydrogenlike atoms, such as the muon or the antiproton.
\end{abstract}

DOI: 10.1103/PhysRevD.104.105009

\section{INTRODUCTION}

Eigenvalues and eigenstates can be defined and derived completely algebraically, without using a Hilbert-space representation of observables as operators. Such a formulation is important in particular in studies of nonassociative algebras that cannot be represented on a Hilbert space. Physical examples can be found mainly in situations in which fractional magnetic charges may be present that do not obey Dirac's quantization condition [1], which can be defined at the level of a nonassociative algebra of observables even though no Hilbert-space representation exists [2-5]. Magnetic monopole charges that obey Dirac's quantization condition are so large that they can easily be ruled out in elementary particles by atomic spectroscopy. While small nonzero magnetic charges may be compatible with observational bounds, they cannot obey the quantization condition and therefore require nonassociative algebras of observables.

Nonassociative products are obtained for magnetic monopoles as follows: In the presence of magnetic monopoles, the magnetic field has nonzero divergence and

\footnotetext{
*bojowald@gravity.psu.edu

†suddhasattwa.brahma@gmail.com

*ubuyukcam@gmail.com

§mvk18@psu.edu
}

Published by the American Physical Society under the terms of the Creative Commons Attribution 4.0 International license. Further distribution of this work must maintain attribution to the author(s) and the published article's title, journal citation, and DOI. Funded by SCOAP. therefore cannot be described by a vector potential. The usual canonical momentum $\hat{\pi}_{i}=\hat{p}_{i}+e \hat{A}_{i}$ of a particle with electric charge $e$ and mass $m$, where $\hat{p}_{i}=m \dot{\hat{x}}_{i}$ is the kinematical momentum, is then unavailable. However, it turns out that the commutator of two kinematical momenta,

$$
\begin{aligned}
{\left[\hat{p}_{j}, \hat{p}_{k}\right] } & =\left[\hat{\pi}_{j}-e \hat{A}_{j}, \hat{\pi}_{k}-e \hat{A}_{k}\right]=i \hbar e\left(\frac{\widehat{\partial A_{k}}}{\partial x_{j}}-\frac{\widehat{\partial A_{j}}}{\partial x_{k}}\right) \\
& =i \hbar e \sum_{l=1}^{3} \epsilon_{j k l} \hat{B}^{l},
\end{aligned}
$$

does not require a vector potential. (The usual bracket $\left[\hat{x}_{j}, \hat{p}_{k}\right]=i \hbar \delta_{j k}$ remains unchanged.) It can therefore be generalized to a point charge moving in the presence of a background magnetic charge, but it is not canonical and not even constant since the magnetic field is position dependent. The Jacobi identity is therefore not guaranteed to hold, and it is indeed violated as the calculation

$$
\begin{gathered}
{\left[\left[\hat{p}_{x}, \hat{p}_{y}\right], \hat{p}_{z}\right]+\left[\left[\hat{p}_{y}, \hat{p}_{z}\right], \hat{p}_{x}\right]+\left[\left[\hat{p}_{z}, \hat{p}_{x}\right], \hat{p}_{y}\right]} \\
=i \hbar e \sum_{j=1}^{3}\left[\hat{B}^{j}, \hat{p}_{j}\right]=-\hbar^{2} e \widehat{\operatorname{div} \vec{B}} \neq 0
\end{gathered}
$$

demonstrates. Since the assumption of an associative product would imply the Jacobi idenity for the commutator, magnetic monopoles are seen to require nonassociative algebras of quantum observables [2-5]. The basic commutators (1) together with an associator determined by (2) can 
be turned into a complete nonassociative algebra by means of $*$-products [6-10].

Purely algebraic derivations that do not make use of specific representations are usually more challenging than standard quantum mechanics, in particular if associativity cannot be assumed. As a consequence, such systems remain incompletely understood, and it remains to be seen whether they can be viable. Nevertheless, it has recently become possible to derive potential physical effects [11] and to use spectral results for new upper bounds on the possible magnetic charge of elementary particles [12]. The present paper presents details of the latter derivation as well as a discussion of new methods that may be useful for further applications.

\section{ASSOCIATIVE ALGEBRA OF THE STANDARD HYDROGEN ATOM}

Modeled by a simple Coulomb potential, the hydrogen atom has the Hamiltonian

$$
H=\frac{1}{2 m}|p|^{2}-\frac{\alpha}{r}
$$

with constant $\alpha$, where $|p|^{2}=p_{x}^{2}+p_{y}^{2}+p_{z}^{2}$ and $r^{2}=$ $x^{2}+y^{2}+z^{2}$ in Cartesian coordinates. As operators, the position and momentum components are subject to the basic commutation relations

$$
\left[\hat{x}, \hat{p}_{x}\right]=\left[\hat{y}, \hat{p}_{y}\right]=\left[\hat{z}, \hat{p}_{z}\right]=i \hbar,
$$

and they are self-adjoint. These conditions define a so-called $*$-algebra, which, together with a quantum Hamiltonian $\hat{H}$, properties of angular momentum, and the virial theorem, will be the only ingredient in our derivation of spectral properties. We will not make use of operators that represent the observables on a Hilbert space of wave functions.

An eigenvalue is a property of an observable in the algebra together with a specific eigenstate. For a derivation of spectral properties we therefore need a notion of states on an algebra, bypassing the introduction of wave functions. Given a $*$-algebra $\mathcal{A}$, a quantum state [13] is defined as a positive linear functional $\omega: \mathcal{A} \rightarrow \mathbb{C}$ from the algebra to the complex numbers, such that $\omega\left(\hat{a}^{*} \hat{a}\right) \geq 0$ for all $a \in \mathcal{A}$. In addition, a state obeys the normalization condition $\omega(\hat{\mathbb{I}})=1$ where $\hat{\mathbb{I}} \in \mathcal{A}$ is the unit. The evaluation $\omega(\hat{a})$ is then the expectation value of $\hat{a} \in \mathcal{A}$, and moments such as $\omega\left(\hat{a}^{n}\right)$ for integer $n$ define a probability distribution for measurements of the observable $a$ if $\hat{a}$ is self-adjoint, $a^{*}=a$. Our aim is to derive properties of eigenvalues $\lambda$ of a quantum Hamiltonian $\hat{H} \in \mathcal{A}$ for hydrogen through a suitable subset of the moment conditions

$$
\omega(\hat{a}(\hat{H}-\lambda))=0 \quad \text { for all } \hat{a} \in \mathcal{A}
$$

We have to find a useful subset of $\hat{a} \in \mathcal{A}$ in order to make this derivation feasible.

\section{A. Subalgebra for spherical symmetry}

Instead of applying standard position and momentum components, spherical symmetry can be used to introduce a promising subset of algebra elements. A subalgebra of certain spherically symmetric elements of $\mathcal{A}$ is generated by the three elements

$$
\hat{r}, \quad \hat{P}=\hat{r}|\hat{p}|^{2}, \quad \hat{Q}=\hat{x} \hat{p}_{x}+\hat{y} \hat{p}_{y}+\hat{z} \hat{p}_{z}-i \hbar .
$$

Linear combinations of these generators form a threedimensional Lie algebra with basic relations

$$
[\hat{r}, \hat{Q}]=i \hbar \hat{r}, \quad[\hat{r}, \hat{P}]=2 i \hbar \hat{Q}, \quad[\hat{Q}, \hat{P}]=i \hbar \hat{P}
$$

isomorphic to $\mathrm{so}(2,1)$. (Closely related algebras have been used for derivations of the hydrogen spectrum in deformation quantization [14-16]. Our application of this algebra follows different methods, and our extension to nonassociative hydrogen in the next section is completely new.) Its Casimir element is given by

$$
\hat{K}:=\frac{1}{2}(\hat{r} \hat{P}+\hat{P} \hat{r})-\hat{Q}^{2} .
$$

Using the definitions (6), $\hat{K}$ turns out to equal the square of angular momentum.

At this point, we can already see the main features of our new derivation, which consists of the following steps in the order of the next three subsections:

(1) A Casimir element such as (8) is a powerful tool because it takes a constant value in a fixed irreducible representation. Physically, our Casimir is not new but identical with the square of angular momentum. Nevertheless, we will examine angular momentum in order to determine which of the standard properties are readily available in a completely algebraic derivation.

(2) The standard hydrogen Hamiltonian $\hat{H}$ is not an element of our linear algebra because the Coulomb potential requires an inverse of $\hat{r}$, and $\hat{P}$ is not the correct kinetic energy. However, the basic variables $\hat{r}$ and $\hat{P}$ are such that the expression $\hat{r} \hat{H}$ is linear in our algebra generators. This observation by itself is not very helpful because $\hat{r} \hat{H}$ and $\hat{H}$ do not have the same eigenvalues. Nevertheless, it is a crucial step in our new strategy, combined with turning the Hamiltonian into a constraint equation: If we start with the constraint $\hat{H}-\lambda=0$, encoded by the algebraic definition (5) of the spectrum, we replace it with the constraint $\hat{r} \hat{H}-\lambda \hat{r}=0$, which is still linear in our basic generators. This step gives rise to several subtleties because we will have to employ 
methods from constrained systems, and even for nonself-adjoint constraints because we need the nonsymmetric ordering $\hat{r} \hat{H}$ in the constraint in order to use $\hat{P}$ in our linear algebra. Once this step is completed, we gain information about solutions of (5).

(3) In the final step, we have to impose positivity of the state used in our solutions of (5). Instead of working directly with the basic positivity condition, we will evaluate uncertainty relations which are derived from positivity. In this general form, there are many different versions of uncertainty relations because they depend on which basic operators one chooses as well as on the polynomial order in which they appear in moments. For our purposes, we do not need a complete analysis but rather have to find a suitable version of uncertainty conditions that gives us useful information about energy eigenvalues. Our main aim is to rule out a certain range of values that could be eigenenergies of nonassociative hydrogen. The specific uncertainty relations we choose turn out to give us an interesting restriction by ruling out a large range of values. If there is another uncertainty relation that rules out more values, it would only strengthen our result without removing the bounds obtained here.

We will first explore the algebra and derive useful identities within it as well as in an extension that includes an inverse $\hat{r}^{-1}$. This inverse does not only appear in the Coulomb potential, where it can be evaded by using the constraint just described, but also, as it turns out, appears in the adjointness relation of $\hat{P}$. Since adjointness relations are essential for positivity conditions or uncertainty relations, we cannot avoid discussing a possible inclusion of $\hat{r}^{-1}$ in the algebra. The identities derived now for this purpose will be used in our main calculation.

The commutators (7) rely on $\hat{P}$ and $\hat{Q}$ being defined in the specific orderings shown in their definition (6), making them not self-adjoint. Completing the definition of a $*$-subalgebra, their adjointness relations can be derived from the basic commutators of Cartesian position and momentum components: In addition to $\hat{r}^{*}=\hat{r}$, we have

$$
\hat{Q}^{*}=\hat{Q}-i \hbar \hat{\mathbb{I}}
$$

and

$$
\hat{P}^{*}=\hat{P}-2 i \hbar \hat{r}^{-1} \hat{Q}=\hat{P}-2 i \hbar \hat{Q} \hat{r}^{-1}-2 \hbar^{2} \hat{r}^{-1} .
$$

At this point, we assume that the subalgebra generated by $\hat{r}$, $\hat{P}$, and $\hat{Q}$ is suitably extended such that it includes an inverse of $\hat{r}$ which, like $\hat{r}$, is also self-adjoint. The adjointness relations imply the conditions

$$
\operatorname{Im} \omega(\hat{Q})=\frac{1}{2 i} \omega\left(\hat{Q}-\hat{Q}^{*}\right)=\frac{1}{2} \hbar
$$

and

$$
\begin{aligned}
\operatorname{Im} \omega(\hat{r} \hat{P}) & =\frac{1}{2 i}\left(\omega(\hat{r} \hat{P})-\omega\left(\hat{P}^{*} \hat{r}\right)\right) \\
& =\frac{1}{2 i}\left(\omega(\hat{P} \hat{r}+2 i \hbar \hat{Q})-\omega\left(\hat{P} \hat{r}-2 i \hbar \hat{Q}-2 \hbar^{2} \hat{\mathbb{I}}\right)\right) \\
& =\hbar \omega\left(\hat{Q}+\hat{Q}^{*}\right)=2 \hbar \operatorname{Re} \omega(\hat{Q})
\end{aligned}
$$

for expectation values in any state $\omega$ on the algebra, in addition to $\operatorname{Im} \omega(\hat{r})=0$.

In deriving (10), we have made use of the commutator

$$
\left[\hat{r},|\hat{p}|^{2}\right]=2 i \hbar \hat{r}^{-1} \hat{Q}=2 i \hbar(\hat{Q}-i \hbar \hat{\mathbb{I}}) \hat{r}^{-1}
$$

which is itself based on the commutator

$$
\left[\hat{r}^{-1}, \hat{Q}\right]=-i \hbar \hat{r}^{-1}
$$

in the second step. These commutators can be computed easily in a position representation of momentum components in $\hat{p}$ and $\hat{Q}$, which then defines the extension of our algebra to one that includes $\hat{r}^{-1}$. Related useful commutators are

$$
\begin{aligned}
{\left[\hat{Q},|\hat{p}|^{2}\right] } & =2 i \hbar \hat{r}^{-1} \hat{P}, \\
{\left[\hat{r}^{-1},|\hat{p}|^{2}\right] } & =2 i \hbar \hat{r}^{-3}(\hat{Q}+i \hbar), \\
{\left[\hat{r}^{-1}, \hat{P}\right] } & =2 i \hbar \hat{r}^{-2}(\hat{Q}+i \hbar),
\end{aligned}
$$

and

$$
\begin{aligned}
{\left[\hat{P}^{*}, P\right]=} & -2 i \hbar \hat{r}^{-1} \hat{Q} \hat{P}^{*}+2 i \hbar \hat{P}(\hat{Q}-i \hbar) \hat{r}^{-1} \\
& +4 \hbar^{2} \hat{r}^{-1} \hat{Q}(\hat{Q}-i \hbar) \hat{r}^{-1} \\
{\left[\hat{P}^{*}, \hat{r}\right]=} & {[\hat{r}, \hat{P}]^{*}=-i \hbar \hat{r} } \\
{\left[\hat{P}^{*}, \hat{Q}\right]=} & -i \hbar \hat{P}^{*}=\left[\hat{P}^{*}, \hat{Q}^{*}\right] .
\end{aligned}
$$

Adjointness relations require us to extend the algebra by an inverse of $\hat{r}$. Nevertheless, we will see that all moments required for a derivation of spectral properties can be derived using relations in the linear algebra because the expectation value $\omega\left(\hat{r}^{-1}\right)$ is related to moments of polynomial expressions by the virial theorem, which states that for any quantum Hamiltonian $\hat{H}=\frac{1}{2} m^{-1} \hat{p}^{2}+\alpha \hat{r}^{n}$ with some integer $n$, the expectation values of kinetic and potential energy in a stationary state $\omega$ are related by

$$
\omega\left(\hat{p}^{2}\right)=n m \alpha \omega\left(\hat{r}^{n}\right) .
$$

Since all energy eigenstates are stationary, the theorem applies in our case. In addition, the eigenvalue condition 
$\omega(\hat{H}-\lambda)=0$, as a special case of (5), implies a second condition for the same energy expectation values:

$$
\frac{1}{2 m} \omega\left(\hat{p}^{2}\right)+\alpha \omega\left(\hat{r}^{n}\right)=\lambda
$$

Therefore,

$$
\omega\left(\hat{p}^{2}\right)=\frac{2 n m \lambda}{n+2}, \quad \alpha \omega\left(\hat{r}^{n}\right)=\frac{2 \lambda}{n+2} .
$$

For the Coulomb potential,

$$
\omega\left(\hat{r}^{-1}\right)=\frac{2 \lambda}{\alpha}
$$

is strictly determined.

The proof of the virial theorem is brief and standard, but it is useful to display the key ingredients to demonstrate that no Hilbert-space representation is required. Since $\omega$ is stationary, we have

$$
\begin{aligned}
0 & =\frac{\mathrm{d} \omega(\hat{Q})}{\mathrm{d} t}=-i \omega\left(\left[\hat{x} \hat{p}_{x}+\hat{y} \hat{p}_{y}+\hat{z} \hat{p}_{z}, \hat{H}\right]\right) \\
& =\omega\left(m^{-1}|\hat{p}|^{2}-n \alpha \hat{r}^{n}\right)
\end{aligned}
$$

using $\left[\hat{p}_{x}, \hat{r}\right]=-i \partial \widehat{r / \partial} x=-i \hat{x} \hat{r}^{-1}$. This result proves the virial theorem not only for standard quantum mechanics but also for nonassociative systems in the presence of magnetic monopoles: While some associativity is applied in computing the commutator in (25), none of the brackets (2) appear that would be modified for nonzero magnetic charge.

\section{B. Angular momentum}

We will use the familiar eigenvalues of angular momentum squared, which equal the eigenvalues of $\hat{K}$ defined in (8). The usual derivation of these eigenvalues is, to a large degree, algebraic, but it relies on applications of ladder operators on wave functions representing angular-momentum eigenstates. Such an application will no longer be available once we turn to nonassociative hydrogen. We therefore provide here a complete algebraic derivation of angular-momentum eigenvalues.

The relevant algebra in this derivation is the enveloping algebra $\mathcal{B}$ of the Lie algebra $\operatorname{su}(2)$, with self-adjoint generators $\hat{J}_{x}, \hat{J}_{y}$, and $\hat{J}_{z}$ such that

$$
\left[\hat{J}_{x}, \hat{J}_{y}\right]=i \hbar \hat{J}_{z}, \quad\left[\hat{J}_{y}, \hat{J}_{z}\right]=i \hbar \hat{J}_{x}, \quad\left[\hat{J}_{z}, \hat{J}_{x}\right]=i \hbar \hat{J}_{y} .
$$

An angular-momentum eigenstate $\omega_{l, \mu}$ with eigenvalue $\iota$ of the square of angular momentum, $\hat{J}^{2}=\hat{J}_{x}^{2}+\hat{J}_{y}^{2}+\hat{J}_{z}^{2}$, and eigenvalue $\mu$ of the $z$-component $\hat{J}_{z}$ is a normalized and positive linear map from $\mathcal{B}$ to the complex numbers which obeys the conditions

$$
\omega_{l, \mu}\left(\hat{a}\left(\hat{J}^{2}-\imath\right)\right)=0 \quad \text { and } \quad \omega_{l, \mu}\left(\hat{a}\left(\hat{J}_{z}-\mu\right)\right)=0
$$

for all $\hat{a} \in \mathcal{B}$.

Although we will not apply the ladder operators $\hat{J}_{ \pm}$to wave functions, defined as usual as

$$
\hat{J}_{ \pm}=\hat{J}_{x} \pm \hat{J}_{y},
$$

they are still useful because they obey the identity

$$
\begin{aligned}
\hat{J}_{-}^{N} \hat{J}_{+}^{N}= & \hat{J}_{-}^{N-1}\left(\hat{J}^{2}-\hat{J}_{z}^{2}-\hat{J}_{z}\right) \hat{J}_{+}^{N-1} \\
= & \hat{J}_{-}^{N-1} \hat{J}_{+}^{N-1}\left(\hat{J}^{2}-\hat{J}_{z}^{2}-\hat{J}_{z}\right) \\
& +\hat{J}_{-}^{N-1}\left[\left(\hat{J}^{2}-\hat{J}_{z}^{2}-\hat{J}_{z}\right), \hat{J}_{+}^{N-1}\right] \\
= & \hat{J}_{-}^{N-1} \hat{J}_{+}^{N-1}\left(\hat{J}^{2}-\hat{J}_{z}^{2}-\hat{J}_{z}-2(N-1) \hat{J}_{z}\right. \\
& \left.-(N-1)^{2}-(N-1)\right)
\end{aligned}
$$

on $\mathcal{B}$ for any positive integer $N$. Similarly,

$$
\begin{aligned}
\hat{J}_{+}^{N} \hat{J}_{-}^{N}= & \hat{J}_{+}^{N-1} \hat{J}_{-}^{N-1}\left(\hat{J}^{2}-\hat{J}_{z}^{2}+\hat{J}_{z}+2(N-1) \hat{J}_{z}\right. \\
& \left.-(N-1)^{2}-(N-1)\right) .
\end{aligned}
$$

Evaluating these identities in an eigenstate, we find

$$
\begin{aligned}
\omega_{\imath, \mu}\left(\hat{J}_{\mp}^{N} \hat{J}_{ \pm}^{N}\right)= & \omega_{l, \mu}\left(\hat{J}_{\mp}^{N-1} \hat{J}_{ \pm}^{N-1}\right) \\
& \times\left(l-(\mu \pm(N-1))^{2} \mp(\mu \pm(N-1))\right) \\
= & \prod_{n=0}^{N-1}\left(l-(\mu \pm n)^{2} \mp(\mu \pm n)\right)
\end{aligned}
$$

by iteration.

Since we have $\hat{J}_{-}^{N} \hat{J}_{+}^{N}=\left(\hat{J}_{+}^{N}\right)^{*}\left(\hat{\boldsymbol{J}}_{+}^{N}\right)$ and $\hat{\boldsymbol{J}}_{+}^{N} \hat{\boldsymbol{J}}_{-}^{N}=\left(\hat{\boldsymbol{J}}_{-}^{N}\right)^{*}\left(\hat{\boldsymbol{J}}_{-}^{N}\right)$, positivity of $\omega_{l, \mu}$ implies

$$
\prod_{n=0}^{N}\left(l-(\mu \pm n)^{2} \pm(\mu \pm n)\right) \geq 0
$$

for all $N \geq 0$. The second term in each factor, $-(\mu \pm n)^{2}$, is a negative square which can grow arbitrarily negative. Therefore, only finitely many factors in the products (30) can be nonzero, such that, for some positive integers $n_{+}$and $n_{-}$, we have

$$
\begin{aligned}
& \imath-\left(\mu+n_{+}\right)^{2}-\left(\mu+n_{-}\right)=0 \quad \text { and } \\
& \imath-\left(\mu-n_{-}\right)^{2}+\left(\mu-n_{-}\right)=0 .
\end{aligned}
$$

Solving these two equations implies that the eigenvalues are of the form

$\iota=\left(\frac{n_{-}+n_{+}}{2}\right)\left(\frac{n_{-}+n_{+}}{2}+1\right)$ and $\mu=\frac{n_{-}-n_{+}}{2}$,

which can be recognized as the familiar eigenvalues in finite-dimensional irreducible representations of $\mathrm{su}(2)$. 


\section{Eigenvalue constraint}

We proceed with our derivation of energy eigenvalues. The Hamiltonian is not polynomial in basic observables of the linear algebra. However, some of the conditions (5) are defined on the linear algebra, provided $\hat{a}$ has at least one factor of $\hat{r}$ on its right. For instance, a single such factor, $\hat{a}=\hat{r}$, replaces the nonpolynomial $\hat{H}-\lambda$ in (5) with the linear expression

$$
\hat{C}:=\hat{r}(\hat{H}-\lambda)=\frac{1}{2 m} \hat{P}-\lambda \hat{r}-\alpha .
$$

A subset of the spectrum conditions (5) can therefore be written in terms of $\hat{C}$ as the constraint equations

$$
\omega(\hat{b} \hat{C})=0 \quad \text { for all } \hat{b} \in \mathcal{A}
$$

These constraints might not be sufficient to obtain the full spectrum based on (5), but any condition on eigenvalues derived from (A17) also applies to the full eigenvalues.

In what follows, we therefore replace the self-adjoint Hamiltonian $\hat{H}$ with a constraint operator $\hat{C}$ that, by definition, is not self-adjoint. Dealing with constraints that are not self-adjoint requires some care. In particular, while a self-adjoint constraint generates a gauge flow in much the same way as a self-adjoint Hamiltonian generates time evolution, there are additional terms in the relationship between the flow and the commutator with $\hat{C}$ when the constraint $\hat{C}$ is not self-adjoint.

For a self-adjoint Hamiltonian $\hat{H}$, a time-dependent state $\omega_{t}$ by definition evolves according to

$$
\begin{aligned}
\frac{\mathrm{d} \omega_{t}(\hat{O})}{\mathrm{d} t} & =\frac{\mathrm{d}}{\mathrm{d} t} \omega_{t}(\exp (i t \hat{H} / \hbar) \hat{O} \exp (-i t \hat{H} / \hbar)) \\
& =\frac{\omega_{t}([\hat{O}, \hat{H}])}{i \hbar}
\end{aligned}
$$

for all $\hat{O} \in \mathcal{A}$. Similarly, defining the gauge flow generated by $\hat{C}$ through the (nonunitary) operator $\hat{F}_{\epsilon}=\exp (-i \epsilon \hat{C} / \hbar)$ a gauge-dependent state $\omega_{\epsilon}$ flows according to

$$
\frac{\mathrm{d} \omega_{\epsilon}(\hat{O})}{\mathrm{d} \epsilon}=\frac{\mathrm{d}}{\mathrm{d} \epsilon} \omega_{\epsilon}\left(\hat{F}_{\epsilon}^{*} \hat{O} \hat{F}_{\epsilon}\right)
$$

because this condition implies that any state solving the constraint equation (A17) is preserved by the flow: We then have

$$
\omega\left(\hat{F}_{\epsilon}^{*} \hat{O} \hat{F}_{\epsilon}\right)=\omega(\hat{O})
$$

for all $\hat{O} \in \mathcal{A}$ if $\omega(\hat{b} \hat{C})=0$ for all $\hat{b} \in \mathcal{A}$. Infinitesimally, applying the flow operator implies a relationship,

$$
\begin{aligned}
\frac{\mathrm{d} \omega_{\epsilon}(\hat{O})}{\mathrm{d} \epsilon} & =\frac{\mathrm{d}}{\mathrm{d} \epsilon} \omega_{\epsilon}\left(\exp \left(i \epsilon \hat{C}^{*} / \hbar\right) \hat{O} \exp (-i \epsilon \hat{C} / \hbar)\right) \\
& =\frac{\omega_{\epsilon}\left(\hat{O} \hat{C}-\hat{C}^{*} \hat{O}\right)}{i \hbar},
\end{aligned}
$$

that is not directly related to the commutator of $\hat{O}$ and $\hat{C}$ because of the presence of a $\hat{C}^{*}$. In our specific case, we can use

$$
\hat{C}^{*}=\hat{C}-\frac{i \hbar}{m} \hat{r}^{-1} \hat{Q}=\hat{C}-\frac{i \hbar}{m} \hat{Q} \hat{r}^{-1}-\frac{\hbar^{2}}{m} \hat{r}^{-1}
$$

and arrive at

$\frac{\mathrm{d} \omega_{\epsilon}(\hat{O})}{\mathrm{d} \epsilon}=\frac{\omega_{\epsilon}([\hat{O}, \hat{C}])}{i \hbar}+\frac{\omega_{\epsilon}\left(\hat{Q} \hat{r}^{-1} \hat{O}\right)}{m}-\frac{i \hbar \omega_{\epsilon}\left(\hat{r}^{-1} \hat{O}\right)}{m}$.

The constraint equations (A17) play the same role as stationarity of eigenstates. Since every energy eigenstate $\omega$ is gauge invariant under the flow generated by $\hat{C}$, (40) implies that

$$
\frac{\omega([\hat{O}, \hat{C}])}{i \hbar}=-\frac{\omega\left((\hat{Q}-i \hbar \hat{\mathbb{I}}) \hat{r}^{-1} \hat{O}\right)}{m}
$$

for any such state. Since $\hat{r}^{-1}$ appears on the right, these equations give us another way to derive moments involving $\hat{r}^{-1}$. For instance, for $\hat{O}=\hat{r}$, we obtain

$$
\omega(\hat{Q})=\frac{\omega([\hat{r}, \hat{P}])}{2 i \hbar}=\frac{m \omega([\hat{r}, \hat{C}])}{i \hbar}=-\omega(\hat{Q}-i \hbar \hat{\mathbb{I}})
$$

from the basic commutators in the first step, the definition of $\hat{C}$ in the second step, and an application of Eq. (41) in the last step. Thus,

$$
\omega(\hat{Q})=\frac{1}{2} i \hbar,
$$

which is consistent with the reality condition (11) and in addition shows that $\operatorname{Re} \omega(\hat{Q})=0$ for stationary states.

In another example, choosing $\hat{O}=\hat{Q}$ and using the basic commutators, as well as (14), implies

$$
\frac{1}{2 m} \omega(\hat{P})+\lambda \omega(\hat{r})+\frac{1}{m} \omega\left(\hat{Q}^{2} \hat{r}^{-1}\right)-\frac{\hbar^{2}}{m} \omega\left(\hat{r}^{-1}\right)=0 .
$$

In this expression, the first term is given by

$$
\frac{1}{2 m} \omega(\hat{P})=\lambda \omega(\hat{r})+\alpha
$$

using the constraint $\omega(\hat{C})=0$. The factor of $\hat{Q}^{2}$ in the third moment can be eliminated by using the Casimir $\hat{K}$, such that 


$$
\begin{aligned}
\hat{Q}^{2} \hat{r}^{-1} & =\left(-\hat{K}+\frac{1}{2}(\hat{r} \hat{P}+\hat{P} \hat{r})\right) \hat{r}^{-1} \\
& =-\hat{K} \hat{r}^{-1}+\frac{1}{2}[\hat{r}, \hat{P}] \hat{r}^{-1}+\hat{P} \\
& =-\hat{K} \hat{r}^{-1}+i \hbar \hat{Q} r^{-1}+\hat{P} .
\end{aligned}
$$

The final appearance of $\hat{Q}$ in the second term of this new expression can be eliminated by applying (41) to $\hat{O}=\hat{\mathbb{I}}$ : $i \hbar \omega\left(\hat{Q} \hat{r}^{-1}\right)=\hbar^{2} \omega\left(\hat{r}^{-1}\right)$. Thus, Eq. (44) implies

$$
3 \alpha+4 \lambda \omega(\hat{r})-\frac{K}{m} \omega\left(\hat{r}^{-1}\right)=0
$$

with an eigenvalue $K=\hbar^{2} \ell(\ell+1)$ of $\hat{K}$ if we assume, as usual, that our eigenstate is simultaneously one of energy and angular momentum and then use the derivation given in Sec. II B. Using the virial theorem to replace $\omega\left(\hat{r}^{-1}\right)=2 \lambda / \alpha$, we obtain

$$
\omega(\hat{r})=-\frac{1}{2} \ell(\ell+1) \frac{\hbar^{2}}{m \alpha}-\frac{3}{4} \frac{\alpha}{\lambda}
$$

for the radius expectation value. This equation gives the correct expression for the $r$-expectation value in all energy eigenstates in terms of the eigenvalue $\lambda$.

\section{Uncertainty relations}

So far, we have not obtained any restriction on the eigenvalues $\lambda$ that may appear in (48). Such restrictions cannot be derived by using only the eigenmoment equation (5). In addition, we have to impose conditions that ensure that $\omega$ is positive (or "normalizable" in quantummechanics lingo). In order to keep the discussion more physically intuitive, we implement positivity through the equivalent conditions implied by uncertainty relations.

\section{General derivation}

To arrive at uncertainty relations, we follow standard results that imply the Cauchy-Schwarz inequality

$$
\omega\left(\hat{a}^{*} \hat{a}\right) \omega\left(\hat{b}^{*} \hat{b}\right) \geq\left|\omega\left(\hat{b}^{*} \hat{a}\right)\right|^{2}
$$

for all $\hat{a}, \hat{b} \in \mathcal{A}$ and any state $\omega$. The proof proceeds by defining a new algebra element $\hat{a}^{\prime}:=\hat{a} \exp \left(-i \arg \omega\left(\hat{b}^{*} \hat{a}\right)\right)$, designed such that $\left|\omega\left(\hat{b}^{*} \hat{a}\right)\right|=\omega\left(\hat{b}^{*} \hat{a}^{\prime}\right)$. This intermediate step allows us to rewrite the positivity condition as

$$
\begin{aligned}
0 & \leq \omega\left(\left(\sqrt{\omega\left(\hat{b}^{*} \hat{b}\right)} \hat{a}^{\prime}-\sqrt{\omega\left(\hat{a}^{\prime *} \hat{a}^{\prime}\right)} \hat{b}\right)^{*}\left(\sqrt{\omega\left(\hat{b}^{*} \hat{b}\right)} \hat{a}^{\prime}-\sqrt{\omega\left(\hat{a}^{\prime *} \hat{a}^{\prime}\right)} \hat{b}\right)\right) \\
& =2 \omega\left(\hat{b}^{*} \hat{b}\right) \omega\left(\hat{a}^{\prime *} \hat{a}^{\prime}\right)-\sqrt{\omega\left(\hat{b}^{*} \hat{b}\right) \omega\left(\hat{a}^{\prime *} \hat{a}^{\prime}\right)}\left(\omega\left(\hat{a}^{\prime *} \hat{b}\right)+\omega\left(\hat{b}^{*} \hat{a}^{\prime}\right)\right) \\
& =2 \omega\left(\hat{b}^{*} \hat{b}\right) \omega\left(\hat{a}^{\prime *} \hat{a}^{\prime}\right)-2 \sqrt{\omega\left(\hat{b}^{*} \hat{b}\right) \omega\left(\hat{a}^{\prime *} \hat{a}^{\prime}\right)}\left|\omega\left(\hat{b}^{*} \hat{a}\right)\right|
\end{aligned}
$$

and to conclude that

$$
\left|\omega\left(\hat{b}^{*} \hat{a}\right)\right| \leq \sqrt{\omega\left(\hat{a}^{*} \hat{a}\right)} \sqrt{\omega\left(\hat{a}^{* *} \hat{a}^{\prime}\right)}=\sqrt{\omega\left(\hat{a}^{*} \hat{a}\right) \omega\left(\hat{b}^{*} \hat{b}\right)} .
$$

Importantly, the result does not require associativity; see also [17].

Choosing $\hat{a}=\hat{O}_{1}-\omega\left(\hat{O}_{1}\right) \hat{\mathbb{I}}$ and $\hat{b}=\hat{O}_{2}-\omega\left(\hat{O}_{2}\right) \hat{\mathbb{I}}$ for self-adjoint $\hat{O}_{1}$ and $\hat{O}_{2}$, we compute the variances $\omega\left(\hat{a}^{*} \hat{a}\right)=\left(\Delta O_{1}\right)^{2}, \omega\left(\hat{b}^{*} \hat{b}\right)=\left(\Delta O_{2}\right)^{2}$ of $O_{1}$ and $O_{2}$, respectively, and $\omega\left(\hat{b}^{*} \hat{a}\right)=\Delta\left(O_{1} O_{2}\right)+\omega\left(\left[\hat{O}_{1}, \hat{O}_{2}\right]\right)$ is related to their covariance $\Delta\left(O_{1} O_{2}\right)$. In this way, the Cauchy-Schwarz inequality implies Heisenberg's uncertainty relation

$$
\left(\Delta O_{1}\right)^{2}\left(\Delta O_{2}\right)^{2}-\Delta\left(O_{1} O_{2}\right)^{2} \geq\left(\sum_{I} C_{12}^{I} \omega\left(\hat{O}_{I}\right)\right)^{2}
$$

for any pair of observales $O_{1}$ and $O_{2}$ whose algebra elements $\hat{O}_{1}$ and $\hat{O}_{2}$ are two of the generators of a linear subalgebra of $\mathcal{A}$ with structure constants $C_{I J}^{K}:\left[\hat{O}_{1}, \hat{O}_{2}\right]=$ $\sum_{I} C_{12}^{I} \hat{O}_{I}$ with a summation range equal to the dimension of the subalgebra. According to (7), we can apply such an uncertainty relation to any pair of the generators $(\hat{r}, \hat{P}, \hat{Q})$. Some of our generators, $\hat{P}$ and $\hat{Q}$, are not self-adjoint. In such a case, according to the derivation shown here where $\left(\Delta O_{1}\right)^{2}$ results from $\omega\left(\hat{a}^{*} \hat{a}\right)$, any variance of a self-adjoint expression should be replaced with the covariance of the algebra element and its adjoint, such as

$$
\Delta(\bar{P} P)=\frac{1}{2} \omega\left(\hat{P}^{*} \hat{P}+\hat{P} \hat{P}^{*}\right)-|\omega(\hat{P})|^{2} .
$$

\section{Relevant moments}

We will apply these (generalized) uncertainty relations to pairs of algebra elements given by $\hat{r}, \hat{P}$, and $\hat{Q}$. An explicit evaluation in terms of the energy eigenvalue requires a derivation of the moments $\omega\left(\hat{r}^{2}\right), \omega\left(\hat{P}^{*} \hat{P}\right), \omega\left(\hat{Q}^{*} \hat{Q}\right)$, $\omega(\hat{r} \hat{P}), \omega(\hat{r} \hat{Q})$, and $\omega\left(\hat{Q}^{*} \hat{P}\right)$. Also here, we can exploit 
(41) for different choices of $\hat{O}$, as well as (A17) for various choices of $\hat{b}$.

We first compute one of the $\hat{Q}$-related moments. First, Eq. (41) evaluated for $\hat{O}=\hat{r}^{2}$ implies

$$
\begin{aligned}
\frac{\omega(\hat{r} \hat{Q}+\hat{Q} \hat{r})}{m} & =\frac{\omega\left(\left[\hat{r}^{2}, \hat{C}\right]\right)}{i \hbar}=-\frac{\omega((\hat{Q}-i \hbar \hat{\mathbb{I}}) \hat{r})}{m} \\
& =\frac{\omega(\hat{r} \hat{Q}-2 \hat{Q} \hat{r})}{m}
\end{aligned}
$$

using the basic commutators (7) in the last step. Therefore, $\omega(\hat{Q} \hat{r})=0 \quad$ and $\quad \omega(\hat{r} \hat{Q})=\omega([\hat{r}, \hat{Q}])=i \hbar \omega(\hat{r})$.

Together with (43), we arrive at

$$
\Delta(r Q)=0 .
$$

For the $\hat{P}$-related moments, we apply (A17) with $\hat{b}$ equal to the three linear generators $\hat{r}, \hat{Q}$, and $\hat{P}$ as well as the adjoint $\hat{P}^{*}$, giving us four equations,

$$
\omega\left(\hat{r}^{2}\right)=\frac{1}{2 m \lambda} \omega(\hat{r} \hat{P})-\frac{\alpha}{\lambda} \omega(\hat{r})
$$

from $\hat{b}=\hat{r}$

$$
\omega(\hat{Q} \hat{P})=i \hbar m \alpha
$$

from $\hat{b}=\hat{Q}$ using $\omega(\hat{Q} \hat{r})=0$ as just derived,

$$
\omega\left(\hat{P}^{2}\right)=2 m \lambda \omega(\hat{P} \hat{r})+2 m \alpha \omega(\hat{P})
$$

from $\hat{b}=\hat{P}$, and

$$
\begin{aligned}
\omega\left(\hat{P}^{*} \hat{P}\right) & =2 m \lambda \omega\left(\hat{P}^{*} \hat{r}\right)+2 m \alpha \omega\left(\hat{P}^{*}\right) \\
& =2 m \lambda \omega(\hat{P} \hat{r})+2 m \alpha \overline{\omega(\hat{P})}-2 m \lambda \hbar^{2}
\end{aligned}
$$

from $\hat{b}=\hat{P}^{*}$ using (10) and (43). We apply gauge invariance (41) to $\hat{O}=\hat{r} \hat{Q}$, such that

$$
\frac{1}{2 m} \omega(\hat{r} \hat{P})+\frac{2}{m} \omega\left(\hat{Q}^{2}\right)+\lambda \omega\left(\hat{r}^{2}\right)+\frac{1}{2 m} \hbar^{2}=0 .
$$

In the last equation, we replace $\hat{Q}^{2}$ with the square of angular momentum and therefore $\hat{K}$, as before. Together with (56) as well as (48), we obtain

$$
\omega\left(\hat{r}^{2}\right)=\frac{3}{4} \frac{\ell(\ell+1) \hbar^{2}}{m \lambda}+\frac{5}{8} \frac{\alpha^{2}}{\lambda^{2}}-\frac{1}{4} \frac{\hbar^{2}}{m \lambda}
$$

which, like (48), is valid for all energy eigenstates in terms of $\lambda$. The remaining equations then allow us to solve for the $\hat{P}$-related moments

$$
\begin{gathered}
\omega(\hat{r} \hat{P})=\frac{1}{2} \ell(\ell+1) \hbar^{2}-\frac{1}{4} m \frac{\alpha^{2}}{\lambda}-\frac{1}{2} \hbar^{2}, \\
\omega(\hat{P} \hat{r})=\frac{1}{2} \ell(\ell+1) \hbar^{2}-\frac{1}{4} m \frac{\alpha^{2}}{\lambda}+\frac{1}{2} \hbar^{2}, \\
\omega\left(\hat{P}^{*} \hat{P}\right)=-\ell(\ell+1) m \lambda \hbar^{2}+\frac{1}{2} m^{2} \alpha^{2}-m \lambda \hbar^{2} .
\end{gathered}
$$

The $\hat{Q}$-related moment $\omega(\hat{Q} \hat{P})=i \hbar m \alpha$ is already determined by (57), which together with (9), (45), and (48) gives

$$
\begin{aligned}
\omega\left(\hat{Q}^{*} \hat{P}\right) & =i \hbar(m \alpha-\omega(\hat{P}))=-i \hbar m(\alpha+2 \lambda \omega(\hat{r})) \\
& =i \hbar\left(\frac{1}{2} m \alpha+\frac{\lambda \ell(\ell+1) \hbar^{2}}{\alpha}\right)
\end{aligned}
$$

which is the second $\hat{Q}$-related moment relevant for uncertainty relations. The final moment, $\omega\left(\hat{Q}^{*} \hat{Q}\right)$, is related to the square of angular momentum by

$$
\begin{aligned}
\omega\left(\hat{Q}^{*} \hat{Q}\right) & =\omega\left(\hat{Q}^{2}\right)-i \hbar \omega(\hat{Q}) \\
& =-\omega(\hat{K})+\frac{1}{2} \omega(\hat{r} \hat{P}+\hat{P} \hat{r})-i \hbar \omega(\hat{Q}) \\
& =-\frac{1}{2} \ell(\ell+1) \hbar^{2}-\frac{m \alpha^{2}}{4 \lambda}+\frac{1}{2} \hbar^{2},
\end{aligned}
$$

using (62), (63), and (43).

We are now in a position to impose positivity of $\omega$. Heisenberg's uncertainty relation for our variables include

$$
(\Delta r)^{2} \Delta(\bar{P} P) \geq|\Delta(r P)+i \hbar \omega(\hat{Q})|^{2}
$$

which is always saturated for our solutions, without restrictions on $\lambda$. In fact, the equality in this statement is implied by the eigenvalue constraint (A17), such that $\omega(\hat{b} \hat{C})=0$ and $\omega\left(\hat{C}^{*} \hat{b}\right)=0$ for any $\hat{b} \in \mathcal{A}$. Since $\hat{C}$ is linear in $\hat{P}$ and $\hat{r}$, any $P$ in the moments in (67) can be replaced by an $r$ as follows:

$$
\begin{aligned}
\Delta(r P)+i \hbar \omega(\hat{Q})= & \frac{1}{2} \omega((\hat{r}-\omega(\hat{r}))(\hat{P}-\omega(\hat{P})) \\
& +(\hat{P}-\omega(\hat{P}))(\hat{r}-\omega(\hat{r})))+\frac{1}{2} \omega([\hat{r}, \hat{P}]) \\
= & \omega((\hat{r}-\omega(\hat{r}))(\hat{P}-\omega(\hat{P}))) \\
\approx & 2 m \lambda \omega\left((\hat{r}-\omega(\hat{r}))^{2}\right) \\
= & 2 m \lambda(\Delta r)^{2}
\end{aligned}
$$

and 


$$
\begin{aligned}
\Delta(\bar{P} P)= & \frac{1}{2} \omega\left(\left(\hat{P}^{*}-\overline{\omega(\hat{r})}\right)(\hat{P}-\omega(\hat{P}))\right. \\
& \left.+(\hat{P}-\omega(\hat{P}))\left(\hat{P}^{*}-\overline{\omega(\hat{r})}\right)\right) \\
\approx & (2 m \lambda)^{2}(\Delta r)^{2}
\end{aligned}
$$

where $\approx$ indicates equality on states obeying the constraint (A17).

The remaining inequalities,

$$
(\Delta r)^{2} \Delta(\bar{Q} Q) \geq\left|\Delta(r Q)+\frac{1}{2} i \hbar \omega(\hat{r})\right|^{2}
$$

and

$$
\Delta(\bar{Q} Q) \Delta(\bar{P} P) \geq\left|\Delta(\bar{Q} P)+\frac{1}{2} i \hbar \omega(\hat{P})\right|^{2}
$$

imply the same condition on solutions of the constraint (A17), but one that nontrivially restricts the values of $\lambda$.

\section{E. Energy eigenvalues}

We evaluate the inequality (70) explicitly, using a simplification implied by (55). For the variances on the left, we have

$$
\begin{aligned}
(\Delta r)^{2}= & \omega\left(\hat{r}^{2}\right)-\omega(\hat{r})^{2} \\
= & \frac{3}{4} \frac{\ell(\ell+1) \hbar^{2}}{m \lambda}+\frac{5}{8} \frac{\alpha^{2}}{\lambda^{2}}-\frac{1}{4} \frac{\hbar^{2}}{m \lambda} \\
& -\left(\frac{1}{2} \ell(\ell+1) \frac{\hbar^{2}}{m \alpha}+\frac{3 \alpha}{4} \frac{\alpha}{\lambda}\right)^{2} \\
= & -\frac{\ell^{2}(\ell+1)^{2} \hbar^{4}}{4 m^{2} \alpha^{2}}+\frac{\alpha^{2}}{16 \lambda^{2}}-\frac{\hbar^{2}}{4 m \lambda}
\end{aligned}
$$

from (48) and (61), and

$$
\begin{aligned}
\Delta(\bar{Q} Q) & =\omega\left(\hat{Q}^{*} \hat{Q}\right)-|\omega(Q)|^{2} \\
& =-\frac{1}{2} \ell(\ell+1) \hbar^{2}-\frac{m \alpha^{2}}{4 \lambda}+\frac{1}{4} \hbar^{2}
\end{aligned}
$$

combining (66) and (43). Subtracting the right-hand side $\frac{1}{4} \hbar^{2} \omega(\hat{r})^{2}$ off (70), using $\Delta(r Q)=0$ according to (55), we obtain the inequality

$$
\begin{aligned}
& \frac{\ell^{3}(\ell+1)^{3} \hbar^{6}}{8 m^{2} \alpha^{2}}+\frac{\ell^{2}(\ell+1)^{2} \hbar^{4}}{16 m^{2} \alpha^{2}}\left(\frac{m \alpha^{2}}{\lambda}-2 \hbar^{2}\right) \\
& -\frac{\ell(\ell+1) \hbar^{2}}{32 m \lambda^{2}}\left(m \alpha^{2}+2 \hbar^{2} \lambda\right) \\
& -\frac{m \alpha^{4}}{64 \lambda^{3}}-\frac{\alpha^{2} \hbar^{2}}{16 \lambda^{2}}-\frac{\hbar^{4}}{16 m \lambda} \geq 0 .
\end{aligned}
$$

Upon multiplication with the positive $\lambda^{2}$, the left-hand side is given by $\lambda^{-1}$ times a polynomial in $\lambda$ of degree three, which can be factorized as

$$
\begin{aligned}
& \frac{(\ell+1)^{2} \hbar^{6}}{8 m^{2} \alpha^{2} \lambda}\left(\ell^{2} \lambda+\frac{1}{2} \frac{m \alpha^{2}}{\hbar^{2}}\right)\left(\lambda+\frac{1}{2} \frac{m \alpha^{2}}{\hbar^{2}(\ell+1)^{2}}\right) \\
& \times\left(\left(\ell^{2}+\ell-1\right) \lambda-\frac{1}{2} \frac{m \alpha^{2}}{\hbar^{2}}\right) \geq 0 .
\end{aligned}
$$

The central parenthesis demonstrates that the inequality is saturated for any energy eigenvalue of the hydrogen problem with maximal angular momentum for a given quantum number $n$, such that $\ell=n-1$, using the standard expression

$$
\lambda_{n}=-\frac{m \alpha^{2}}{2 \hbar^{2} n^{2}}=-\frac{m \alpha^{2}}{2 \hbar^{2}(\ell+1)^{2}} .
$$

Each degenerate energy level therefore contains a state that saturates an uncertainty relation, (70), even if it is highly excited. This surprising result extends an observation made in $[12,18]$ for the harmonic oscillator to the hydrogen problem.

\section{F. Spectral conditions from uncertainty relations}

The saturation result makes use of the known formula for energy eigenvalues of the hydrogen problem. Keeping in mind our aim to apply algebraic methods to the nonassociative generalization of the problem in the presence of small magnetic charges, we are interested also in an independent derivation of spectral properties directly from the inequality (75). To this end, we first note that the lefthand side of this inequality approaches positive infinity for $\lambda \rightarrow-\infty$, while it has negative roots. In order to demonstrate this result it is useful to split the discussion into two case, $\ell=0$ and $\ell>0$. In the first case, we can rewrite the inequality as

$$
-\frac{\hbar^{4}}{16 m^{2} \lambda}\left(\lambda+\frac{1}{2} \frac{m \alpha^{2}}{\hbar^{2}}\right)^{2} \geq 0
$$

which eliminates all positive $\lambda$ (where we have a continuous spectrum and therefore no normalizable states $\omega$ ), and distinguishes the ground-state energy $\lambda=-\frac{1}{2} m \alpha^{2} / \hbar^{2}$ through a saturation condition. In the second case, the inequality written as

$$
\begin{aligned}
& \frac{\ell^{2}(\ell+1)^{2} \hbar^{6}}{8 m^{2} \alpha^{2}}\left(\lambda+\frac{1}{2} \frac{m \alpha^{2}}{\hbar^{2} \ell^{2}}\right)\left(\lambda+\frac{1}{2} \frac{m \alpha^{2}}{\hbar^{2}(\ell+1)^{2}}\right) \\
& \times\left(\ell^{2}+\ell-1-\frac{1}{2} \frac{m \alpha^{2}}{\hbar^{2} \lambda}\right) \geq 0
\end{aligned}
$$


has a final parenthesis which is always positive for negative $\lambda$. Therefore, it rules out any values of $\lambda$ between the two roots given by the first two parentheses,

$$
\lambda_{1}=-\frac{1}{2} \frac{m \alpha^{2}}{\hbar^{2} \ell^{2}} \quad \text { and } \quad \lambda_{2}=-\frac{1}{2} \frac{m \alpha^{2}}{\hbar^{2}(\ell+1)^{2}}
$$

where $\lambda_{1}<\lambda_{2}$. All intervals between the known degenerate eigenvalues are therefore eliminated. (An alternative derivation of this result not based on uncertainty relations is given in the Appendix A.)

\section{NONASSOCIATIVE HYDROGEN WITH SMALL MAGNETIC CHARGE}

A nonassociative monopole algebra is not uniquely determined by basic commutators and associators such as (1) for a monopole system. Different versions can be classified via suitable star products that determine noncommutative and nonassociative compositions of the basic position and momentum variables as formal power series in $\hbar$. To leading order, a direct calculation demonstrates that the commutators within the subset $\{\hat{r}, \hat{P}, \hat{Q}\}$ remain unchanged compared with the associative case, provided the background magnetic field obeys the condition

$$
\vec{r} \times \vec{B}=0
$$

In this case, therefore, corrections to our preceding results are at most perturbative in $\hbar$ multiplied by a number, such as the magnetic charge, that characterizes the strength of the magnetic field which appears in nontrivial commutators and associators of a monopole star product. Since we will be interested in weak magnetic charges, these corrections will be small.

In order to determine how the magnetic charge appears, we further evaluate condition (80). In general, it implies that $\vec{B}(\vec{r})=b(\vec{r}) \vec{r}$ with some function $b(\vec{r})$. In the static case, we need $\nabla \times \vec{B}=0$, which is fulfilled if and only if $b(r)$ is spherically symmetric. A monopole density $\mu(r)=$ $\nabla \cdot \vec{B}$ then requires

$$
b(r)=\frac{g(r)}{4 \pi r^{3}}
$$

with the magnetic charge

$$
g(r)=4 \pi \int^{r} \mu(\tilde{r}) \tilde{r}^{2} \mathrm{~d} \tilde{r}
$$

enclosed in a sphere of radius $r$. For a single monopole at $r=0, g(r)$ is constant, while $g(r)$ depends on $r$ for a constant monopole density. We will assume that $g(r)=g$ is constant, which combined with the standard Coulomb potential implies that the hydrogen nucleus has magnetic charge $g$.

Given the magnetic field of a single monopole with magnetic charge $g$, according to $[19,20]$ the shifted angular momentum components $\hat{L}_{j}^{\prime}=\hat{L}_{j}+e g \hat{x}_{j} \hat{r}^{-1}$ satisfy the usual commutators of angular momentum and therefore have the familiar spectrum. The Casimir of the algebra generated by $\hat{r}, \hat{P}$, and $\hat{Q}$ is still equal to $\hat{K}=\hat{L}^{2}$, but in terms of the modified angular momentum, it has an extra term:

$$
\hat{K}=\hat{L}^{2}=\hat{L}^{\prime 2}-e^{2} g^{2} \hat{\mathbb{I}} .
$$

(A monopole density with nonconstant $g, \hat{K}$, and $\hat{L}^{\prime 2}$ cannot be diagonalized simultaneously and an independent method would have to be used to find eigenvalues of $\hat{K}$.)

For a single monopole at the center, the spectrum of $\hat{K}$, according to (83), has a simple constant shift compared with the spectrum of $\hat{L}^{\prime 2}$, which is known to break the degeneracy of the energy spectrum for magnetic monopoles that obey Dirac's quantization condition [21]. This condition, $e g=\frac{1}{2} \hbar$, implies a large value of the smallest nonzero magnetic charge because the electric fine structure constant is small. Dirac monopoles in a hydrogen nucleus would therefore be large perturbations that strongly modify the energy spectrum. They can easily be ruled out by standard spectroscopy. Dirac's quantization condition can be violated in nonassociative quantum mechanics. Magnetic charges can then be small and might modify the energy spectrum sufficiently weakly to be phenomenologically viable. However, a derivation of eigenvalues in the nonassociative setting remained impossible for decades. Our methods from the preceding section can now be applied to this question.

We will focus on a range of small magnetic charges $g$ characterized by the condition $0<e g / \hbar<\frac{1}{2}$. As already noted, the commutators (7), the virial theorem, and the Cauchy-Schwarz inequality all hold for a nonassociative monopole algebra, at least up to higher-order terms in the star product. Specifically, corrections from the associator (2) or the commutator to real quantities are of the order $\hbar^{2} e g$ or smaller. Second-order corrections in $\hbar$ and $e g$ are therefore insensitive to the specific star product. To within this order, the only assumption that need be modified in our previous derivation of uncertainty relations is the spectrum of $\hat{K}$, which is no longer equal to the square of angular momentum but instead has the eigenvalues

$$
K_{\ell}=\ell(\ell+1) \hbar^{2}-e^{2} g^{2} .
$$

It is convenient to parametrize the shift by replacing $\ell$ with a noninteger quantum number 


$$
\tilde{\ell}=\sqrt{\left(\ell+\frac{1}{2}\right)^{2}-\frac{e^{2} g^{2}}{\hbar^{2}}}-\frac{1}{2} .
$$

Substituting $\tilde{\ell}$ for $\ell$ in (75) then gives us conditions on energy eigenvalues of nonassociative hydrogen. (Saturation conditions indeed give us correct eigenvalues according to [21], but since the usual degeneracy is broken, they do not give us the full spectrum.)

The range of $\ell$ is bounded by the fact that $\hat{K}$ is a positive operator (the components $\hat{L}_{i}$ being Hermitian [19,20]), such that the eigenvalues (84) cannot be negative. This condition rules out the quantum number $\ell=0$, but for small magnetic charges the next possible value, $\ell=1 / 2$, is allowed. We will assume this value for the ground state because (75) tells us that the smallest root of this equation is proportional to $-1 / \ell^{2}$. The minimum energy eigenvalue is therefore obtained for the smallest possible $\ell$. This value of $\ell$ implies

$$
\tilde{\ell}=\sqrt{1-\frac{e^{2} g^{2}}{\hbar^{2}}}-\frac{1}{2}
$$

which lies in the range

$$
\frac{1}{2}(\sqrt{3}-1)<\tilde{\ell}<\frac{1}{2} .
$$

Since $\tilde{\ell}=0$ is not possible, the uncertainty relation always rules out a range of energy eigenvalues between

$$
\lambda_{1}=-\frac{1}{2} \frac{m \alpha^{2}}{\hbar^{2} \tilde{\ell}^{2}}
$$

and

$$
\lambda_{2}=-\frac{1}{2} \frac{m \alpha^{2}}{\hbar^{2}(\tilde{\ell}+1)^{2}} .
$$

For any $\tilde{\ell}$ in the range (87), $\tilde{\ell}<1$ while $\tilde{\ell}+1>1$. Therefore, a certain nonempty range around the usual hydrogen ground-state energy $-\frac{1}{2} m \alpha^{2} / \hbar^{2}$ is ruled out for any value of a small magnetic charge. We conclude that even a small magnetic charge would strongly modify the usual hydrogen spectrum and be incompatible with spectroscopic data. This strict exclusion is possible because the positivity of $\hat{K}$ implies a discontinuity of energy eigenvalues as functions of the magnetic charge $g$ at $g=0$.

\section{CONCLUSIONS}

Our derivations have produced the first results about spectral properties in a system of nonassociative quantum mechanics. In particular, we have been able to demonstrate a discontinuity in the ground-state energy of hydrogen as a function of the magnetic charge of the nucleus. Addressing this question requires a continuous range of the magnetic charge around zero, which cannot be modeled by an associative treatment with Dirac monopoles for which the magnetic charge is quantized. Nonassociative quantum mechanics is able to describe fractional magnetic charges of any value and is therefore a suitable setting for our question.

A Hilbert-space representation of an algebra by operators acting on wave functions is by necessity associative because for any $\psi$ in the Hilbert space and operators $\hat{A}$, $\hat{B}$, and $C$ we have

$$
(\hat{A} \hat{B}) \hat{C} \psi=\hat{A} \hat{B} \psi^{\prime}=\hat{A}\left(\hat{B} \psi^{\prime}\right)=\hat{A}(\hat{B} \hat{C}) \psi,
$$

defining $\psi^{\prime}=\hat{C} \psi$ in an intermediate step. Nonassociative quantum mechanics can therefore not be represented on a Hilbert space, necessitating a purely algebraic derivation of properties of expectation values, moments, and eigenvalues. That such an algebraic treatment can indeed be used to derive a complete spectrum is demonstrated in [12,18], in this case for the (associative) harmonic oscillator as a proof of principle. The algebraic treatment relies on uncertainty relations in order to impose positivity of states, replacing the more common normalizability conditions of Hilbertspace treatments. The new methods are therefore well suited to finding unexpected saturation properties of eigenstates, even excited ones. As a new result of $[12,18]$, every eigenstate of the harmonic oscillator saturates a suitable uncertainty relation. Saturation results even extend to eigenstates of anharmonic systems in perturbative treatments.

Our application of related methods to nonassociative hydrogen in the present paper have not resulted yet in a full energy spectrum because we focused on the ground state, deriving only one uncertainty relation explicitly. Nevertheless, a saturation result has been found for this state, indicating that the behavior seen in harmonic models might be extendable also to excited states of hydrogen. However, the dynamical algebra of hydrogen is more involved than the canonical algebra applicable to the harmonic oscillator, making a generic treatment of saturation results for hydrogen more complicated.

Our extension to nonassociative hydrogen relied on several fortuitous algebraic properties of standard hydrogen that are not affected by introducing nonassociativity of monopole type, given by a commutator (1) of kinematical momentum components with a magnetic field generated by a pointlike magnetic charge. For other nonassociative algebras, or even a monopole algebra with a continuous magnetic charge distribution, the eigenvalue problem cannot yet be solved, presenting a challenging mathematical problem.

Our specific physical result demonstrates that the pursuit of these mathematical questions is worthwhile. We have found that the ground-state energy of hydrogen with a small 
magnetic nuclear charge $g$ is significantly displaced from the usual value due to a discontinuity, even for infinitesimally small magnetic charge. Spectroscopy is therefore very sensitive to introducing a magnetic charge. In order to produce an upper bound on $g$ consistent with observational data, we may, following [12], wash out the discontinuity implied by positivity of the nonassociative angular momentum $K$ because the eigenvalues of angular momentum squared are determined only within some $\delta L^{2}$ from a purely phenomenological viewpoint. In addition, a fundamental uncertainty in angular momentum could also be caused by an extended magnetic charge distribution in the nucleus, which would imply that $\hat{K}$ and $\hat{L}^{\prime 2}$ no longer commute.

As an estimate of this uncertainty, we may use the value $5 \times 10^{-19}$ given as the accuracy of recent atomic clocks [22], which rely on sharp spectral lines that would be affected by the same uncertainty $\delta L^{2}$ if angular momentum is not sharp. The inequality $K \geq 0$ for eigenvalues of $\hat{K}$, which must always hold because $\hat{K}$ is defined as a positive operator, then implies an upper bound

$$
\begin{aligned}
g & \leq \frac{4 \pi \epsilon_{0} \sqrt{\delta L^{2}} c^{2}}{e} \approx 4.7 \times 10^{-18} \mathrm{Am} \\
& =1.4 \times 10^{-9} g_{\text {Dirac }}
\end{aligned}
$$

for the magnetic charge, written here in SI units. This upper bound is a small fraction of $g_{\text {Dirac }}$, the smallest nonzero magnetic charge allowed by Dirac's quantization condition in an associative treatment.

Magnetic charges of elementary particles have been bounded by various means. Using the proton as an example, interpreted here as the nucleus of hydrogen, our bound is not as strong as those found based on the total magnetic charge of a large number of nucleons in macroscopic objects [23,24]. The large number of nucleons in macroscopic objects implies a strong magnification factor in the latter studies if their magnetic charges add up. However, this method is not available for those elementary particles that cannot be combined in stable macroscopic objects, such as unstable particles or antimatter. Some of them can nevertheless be used as substitutes of the nuclear proton in hydrogenlike atoms, with precision spectroscopic data being available in some cases such as muonium [25] or antihydrogen [26,27]. For instance, muonium spectroscopy with a current accuracy of about $10^{-9}$ gives us an upper bound on the muon's magnetic charge of $g_{\text {muon }} \leq 4.5 \times 10^{-5} g_{\text {Dirac }}$, which is better than available upper bounds based on other methods.

\section{ACKNOWLEDGMENTS}

This work was supported in part by NSF Grant No. PHY1912168. S. B. is supported in part by the NSERC (funding reference No. CITA 490888-16) through a CITA National Fellowship and by a McGill Space Institute fellowship.

\section{APPENDIX A: ALGEBRAIC DERIVATION OF THE ASSOCIATIVE HYDROGEN SPECTRUM}

It is instructive to derive the standard energy spectrum of an electric charge in a Coulomb potential by algebraic means, using the same subalgebra of observables generated by (6) as employed in the main text but imposing positivity of states not through uncertainty relations but, more indirectly, through convergence properties of certain expectation values expressed as power series. This derivation more closely resembles the standard derivation based on convergence properties of norms of wave functions, but it is still fully algebraic. However, it does not give rise to new saturation conditions of uncertainty relations, and it is more difficult to extend it to nonassociative systems.

In addition to the basic commutators (7), we will make use of

$$
[\hat{r}, \hat{C}]=\frac{i \hbar}{m} Q \quad \text { and } \quad[\hat{Q}, \hat{C}]=i \hbar\left(\frac{1}{2 m} \hat{P}+\lambda \hat{r}\right)
$$

with the constraint $\hat{C}$ defined in (33), as well as the expectation-value equation

$$
\omega(\hat{b} \hat{P})=2 m \omega(\hat{b}(\lambda \hat{r}+\alpha))
$$

for any $\hat{b} \in \mathcal{A}$, implied by the eigenvalue constraint (A17). We will apply the invariance condition (41) in various ways, and use the operator (8) in the form

$$
\hat{K}=\hat{r} \hat{P}-i \hbar \hat{Q}-\hat{Q}^{2}
$$

\section{Kramer's relation}

Our first step is the algebraic derivation of a recurrence relation for expectation values of integer powers of $\hat{r}$ in energy eigenstates of hydrogen, known as Kramer's relation. To this end, we derive the commutators

$$
\begin{aligned}
& {\left[\hat{r}^{n}, \hat{Q}\right]=i \hbar n \hat{r}^{n},} \\
& {\left[\hat{r}^{n}, \hat{P}\right]=2 i n \hbar \hat{r}^{n-1} \hat{Q}+\hbar^{2} n(n-1) \hat{r}^{n-1}}
\end{aligned}
$$

for integer $n$, using induction and being careful with taking commutators of powers because $[\hat{a},[\hat{a}, \hat{b}]]=0$ does not always hold for $\hat{a}, \hat{b} \in \mathcal{A}$. 
Second, invariance applied to $\hat{O}=m \hat{r}^{s}$ takes the form

$$
\begin{aligned}
0 & =\frac{m}{i \hbar} \omega\left(\left[\hat{r}^{s}, \hat{C}\right]+(\hat{Q}-i \hbar) \hat{r}^{s-1}\right) \\
& =\frac{1}{2 i \hbar} \omega\left(\left[\hat{r}^{s}, \hat{P}\right]+\left[(\hat{Q}-i \hbar), \hat{r}^{s-1}\right]+\hat{r}^{s-1}(\hat{Q}-i \hbar)\right) \\
& =s \omega\left(\hat{r}^{s-1} \hat{Q}\right)-\frac{i \hbar}{2} s(s-1) \omega\left(\hat{r}^{n-1}\right)-(s-1) i \hbar \omega\left(\hat{r}^{s-1}\right)+\omega\left(\hat{r}^{s-1}(\hat{Q}-i \hbar)\right) \\
& =\frac{s+1}{2} \omega\left(\hat{r}^{s-1}(2 \hat{Q}-i s \hbar)\right)
\end{aligned}
$$

such that

$$
\omega\left(\hat{r}^{s-1} \hat{Q}\right)=\frac{1}{2} i \hbar s \omega\left(\hat{r}^{s-1}\right)
$$

Using this result, invariance applied to $\hat{O}=m \hat{r}^{s} \hat{Q}$ leads to

$$
\begin{aligned}
0 & =\frac{m}{i \hbar} \omega\left(\left[\hat{r}^{s} \hat{Q}, \hat{C}\right]+(\hat{Q}-i \hbar) \hat{r}^{s-1} \hat{Q}\right) \\
& =\frac{1}{2 i \hbar} \omega\left(\left[\hat{r}^{s}, \hat{P}\right] \hat{Q}\right)+\frac{m}{i \hbar} \omega\left(\hat{r}^{s}[\hat{Q}, \hat{C}]\right)+\omega\left(\left[(\hat{Q}-i \hbar), \hat{r}^{s-1}\right] \hat{Q}\right)+\omega\left(\hat{r}^{s-1}(\hat{Q}-i \hbar) \hat{Q}\right) \\
& =s \omega\left(\hat{r}^{s-1} \hat{Q}^{2}\right)-\frac{i \hbar}{2} s(s-1) \omega\left(\hat{r}^{s-1} \hat{Q}\right)+\frac{1}{2} \omega\left(\hat{r}^{s}(\hat{P}+2 m \lambda \hat{r})\right)-i \hbar(s-1) \omega\left(\hat{r}^{s-1} \hat{Q}\right)+\omega\left(\hat{r}^{s-1}(\hat{Q}-i \hbar) \hat{Q}\right) \\
& =(s+1) \omega\left(\hat{r}^{s-1} \hat{Q}^{2}\right)+\frac{1}{2} \omega\left(\hat{r}^{s} \hat{P}\right)+m \lambda \omega\left(\hat{r}^{s+1}\right)-i \hbar \frac{s(s+1)}{2} \omega\left(\hat{r}^{s-1} \hat{Q}\right) \\
& =-(s+1) \omega\left(\hat{K} \hat{r}^{s-1}\right)+(s+3 / 2) \omega\left(\hat{r}^{s} \hat{P}\right)+m \lambda \omega\left(\hat{r}^{s+1}\right)-i \hbar \frac{(s+2)(s+1)}{2} \omega\left(\hat{r}^{s-1} \hat{Q}\right) \\
& =-(s+1) \omega\left(\hat{K} \hat{r}^{s-1}\right)+(s+3 / 2) \omega\left(\hat{r}^{s} \hat{P}\right)+m \lambda \omega\left(\hat{r}^{s+1}\right)+\hbar^{2} \frac{(s+2)(s+1) s}{4} \omega\left(\hat{r}^{s-1}\right)
\end{aligned}
$$

Equation (A2) then implies Kramer's relation

$$
0=\hbar^{2}(s+1)\left(\frac{s(s+2)}{4}-\ell(\ell+1)\right) \omega\left(\hat{r}^{s-1}\right)+(2 s+3) m \alpha \omega\left(\hat{r}^{s}\right)+2(s+2) m \lambda \omega\left(\hat{r}^{s+1}\right)
$$

after inserting the standard angular-momentum eigenvalues of $\hat{K}$. Incidentally, invariance applied to $\hat{O}=\hat{r}^{s} \hat{P}$ results in an identity:

$$
\begin{aligned}
\frac{m}{i \hbar} \omega\left(\left[\hat{r}^{s} \hat{P}, \hat{C}\right]\right)+\omega\left((\hat{Q}-i \hbar) \hat{r}^{s-1} \hat{P}\right)= & \frac{1}{2 i \hbar} \omega\left(\left[\hat{r}^{s}, \hat{P}\right] \hat{P}\right)+\frac{m}{i \hbar} \omega\left(\hat{r}^{s}[\hat{P}, \hat{C}]\right)+\omega\left(\left[(\hat{Q}-i \hbar), \hat{r}^{s-1}\right] \hat{P}\right)+\omega\left(\hat{r}^{s-1}(\hat{Q}-i \hbar) \hat{P}\right) \\
= & 2 m(s+1) \omega\left(\hat{r}^{s-1} \hat{Q}(\lambda \hat{r}+\alpha)\right)-i \hbar m s(s+1) \omega\left(\hat{r}^{s-1}(\lambda \hat{r}+\alpha)\right)+\omega\left(\hat{r}^{s}(2 m \lambda \hat{Q})\right) \\
= & 2 m \alpha(s+1) \omega\left(\hat{r}^{s-1} \hat{Q}\right)+2 m \lambda(s+1) \omega\left(\hat{r}^{s}(\hat{Q}-i \hbar)\right)-i \hbar m s(s+1) \lambda \omega\left(\hat{r}^{s}\right) \\
& -i \hbar m \alpha s(s+1) \omega\left(\hat{r}^{s-1}\right)+2 m \lambda \omega\left(\hat{r}^{s} \hat{Q}\right)=0
\end{aligned}
$$

upon using (A5).

\section{Spectrum}

Equipped with Kramer's relation, which we first shift down by one unit in $s$,

$$
0=\hbar^{2} s\left(\frac{s^{2}-1}{4}-\ell(\ell+1)\right) \omega\left(\hat{r}^{s-2}\right)+(2 s+1) m \alpha \omega\left(\hat{r}^{s-1}\right)+2(s+1) m \lambda \omega\left(\hat{r}^{s}\right)
$$


we can now set up a new recurrence relation. We first generalize Kramer's relation to

$$
\begin{aligned}
0= & \frac{\hbar^{2}}{4} \omega\left((\hat{r} f(\hat{r}))^{\prime \prime \prime}\right)-\hbar^{2} \ell(\ell+1) \omega\left(\hat{r}^{-1} f^{\prime}(\hat{r})\right) \\
& +m \alpha \omega\left(2 f^{\prime}(\hat{r})+\hat{r}^{-1} f(\hat{r})\right)+2 m \lambda \omega\left((\hat{r} f(\hat{r}))^{\prime}\right)
\end{aligned}
$$

for any analytic function $f$, where derivatives of analytic functions of $\hat{r}$ are interpreted in the sense of formal power series.

Specializing $f(\hat{r})$ to $f_{s, k}(\hat{r})=\hat{r}^{s} e^{-k \hat{r}}$ and defining

$$
\kappa_{s}(k, \lambda)=\omega\left(\hat{r}^{s} e^{-k \hat{r}}\right)
$$

then gives

$$
\begin{aligned}
0= & \hbar^{2} s\left(-1-4 \ell(1+\ell)+s^{2}\right) \kappa_{s-2}(k, \lambda) \\
& +\left(h^{2} k(4 \ell(1+\ell)-3 s(1+s))\right. \\
& +4 m(1+2 s) \alpha) \kappa_{s-1}(k, \lambda) \\
& +\left(3 \hbar^{2} k^{2}(1+s)+8 m(\lambda(1+s)-k \alpha)\right) \kappa_{s}(k, \lambda) \\
& -k\left(8 m \lambda+k^{2} \hbar^{2}\right) \kappa_{s+1}(k, \lambda) .
\end{aligned}
$$

Again shifting $s$ by defining $L_{s}(k, \lambda)=\kappa_{s-2}(k, \lambda)$, we rewrite the previous relation as the third-order linear differential equation

$$
\begin{aligned}
0= & \left(\hbar^{2} s\left(-1-4 \ell(1+\ell)+s^{2}\right)\right. \\
& -\left(h^{2} k(4 \ell(1+\ell)-3 s(1+s))+4 m(1+2 s) \alpha\right) \partial_{k} \\
& +\left(3 \hbar^{2} k^{2}(1+s)+8 m(\lambda(1+s)-k \alpha)\right) \partial_{k}^{2} \\
& \left.+k\left(8 m \lambda+k^{2} \hbar^{2}\right) \partial_{k}^{3}\right) L_{s}(k, \lambda) .
\end{aligned}
$$

Since our $f_{s, k}(\hat{r})$ is a bounded operator for $k>0$ and $s \geq 0$ with $\lim _{k \rightarrow \infty} f(\hat{r})=\hat{0}$, any state should be such that $L_{s}(k, \lambda)$ is well defined for all $k>0$ and $s \geq 0$ with $\lim _{k \rightarrow \infty} L_{s}(k, \lambda)=0$ for all $\lambda$. We also know that $L_{s}(k, \lambda)$ is well defined for energy eigenstates at $k=0$ as long as $s \geq 0$ is integer, because Kramer's relation together with the virial theorem provides finite numbers for expectation values of positive integer powers of $\hat{r}$. Under these conditions, we can perform a Laplace-like transformation and write

$$
\begin{aligned}
L_{s}(k, \lambda) & =\int_{0}^{\infty} a_{s, \lambda}(b, d)(k+d(s, \lambda))^{-b} \mathrm{~d} b \\
& =\sum_{n=0}^{\infty} \int_{0}^{1} a_{s, \lambda}(b+n, d)(k+d(s, \lambda))^{-n-b} \mathrm{~d} b .
\end{aligned}
$$

In the first line, $a_{s, \lambda}(b, d)$ may be seen as the inverse Laplace transform of $L_{s}\left(e^{t}-d(s, \lambda)\right)$ with respect to $t$. As we will see, it is convenient to introduce a free displacement $d(s, \lambda)$ on which the coefficients $a_{n, \lambda}$ will in general depend.

For further convenience, we now drop the explicit dependences on $s$ and $\lambda$ from our notation. Comparing coefficients of the expansion (A12) inserted in (A11), we obtain the recurrence relation

$$
\begin{aligned}
& C_{3} a(b+n-3)+C_{2} a(b+n-2)+C_{1} a(b+n-1) \\
& \quad+C_{0} a(b+n)=0
\end{aligned}
$$

with

$$
C_{3}=d(b+n-3)(b+n-2)(b+n-1)\left(d^{2} \hbar^{2}+8 m \lambda\right),
$$

$$
\begin{aligned}
C_{2}= & (b+n-2)(b+n-1)\left(-3 d^{2} \hbar^{2}(b+n-1-s)\right. \\
& +8 d m \alpha-8 m(b+n-1-s) \lambda), \\
C_{1}= & (b+n-1)\left(3 d \hbar^{2}(b+n)(b+n+1)\right. \\
& +d \hbar^{2}(-4 \ell(1+\ell)+3 s(1+s)), \\
& \left.+(b+n)\left(-6 d \hbar^{2}(1+s)-8 m \alpha\right)+4 m \alpha(1+2 s)\right),
\end{aligned}
$$

$C_{0}=-h^{2}(b+n-s)\left((b+n-s)^{2}-(2 \ell+1)^{2}\right)$.

By definition, the support of $a$ as a function of $b$ is bounded from below. If for a given solution $n_{\min }$ is the smallest integer such that $a\left(b+n_{\min }\right) \neq 0$ while $a(b+n)=0$ for $n<n_{\min }$, the expression (A17) shows that $n_{\min }+b-s=0$ or $\left|n_{\min }+b-s\right|-|2 \ell+1|=0$. Using the fact that $\ell$ is an integer (since we are for now assuming the absence of a magnetic charge), $b$ must be an integer. This result shows that $L_{s}(k, \lambda)$ allows an expansion as a Laurent series of the form

$$
L_{s}(k, \lambda)=\sum_{n=0}^{\infty} A_{s, \lambda}(n)(k+d(s, \lambda))^{-n}
$$

[The original coefficients $a_{s, \lambda}(b, d)$ introduced in (A12) are proportional to a Dirac comb of delta functions of $b$ supported on the integers.]

The recurrence relation for $A_{s, \lambda}(n)$ can easily be obtained from (A13) by absorbing $b$ in $n$, ignoring the shift by $b$. The relation can be simplified further by making the choice $d=\sqrt{-8 m \lambda} / \hbar$ for a given $\lambda$, such that the lowest-order term (at order $n-3$ ) drops out of the recurrence. We also choose $s=2 \ell+2$ and obtain 


$$
\begin{aligned}
0= & 2 d(n-2)\left(d \hbar^{2}(3+2 \ell-n)+4 m \alpha\right) A(n-2) \\
& +\left(d \hbar^{2}\left(8 \ell^{2}+\ell(26-12 n)+3(n-3)(n-2)\right)\right. \\
& +4 m \alpha(5+4 \ell-2 n)) A(n-1) \\
& -\hbar^{2}(n-3-4 \ell)(n-2-2 l) A(n)
\end{aligned}
$$

after factoring out $b+n-1$. For very large $n$ of either sign, this recursion takes the form $A(n)-3 d A(n-1)+$ $2 d^{2} A(n-2)=0$, such that any nonzero asymptotic $A_{n}$ behaves either as $d^{n}$ or $(2 d)^{n}$. However, these options would introduce a pole for $L_{s}(k, \lambda)$, either at $k=0$ or $k=d>0$, which cannot happen for well-defined states. Therefore, only finitely many $A(n)$ can be nonzero. According to the $A(n)$-term in (A19), there is an $N_{1}$ such that $A(n)=0$ for $n<N_{1}$ because $\ell$ is an integer.

For the range of $n$ where $A(n) \neq 0$ to be bounded from above, the first coefficient in (A19) requires

$$
d=\frac{4 m \alpha}{\hbar^{2} \nu}
$$

with some positive integer $\nu$. Inserting this expression, we obtain

$$
\begin{aligned}
0= & 2(n+2 \ell)(n-1-\nu) c_{n-2} \\
& -(n(3 n-3-2 \nu)+\nu-4 \ell(1+\ell)) c_{n-1} \\
& +n(n-1-2 \ell) c_{n}
\end{aligned}
$$

where

$$
c_{n}=d^{-n} A_{n+2 \ell+2} .
$$

There is one final condition: as all these sequences are linear with recurrence relations that have integer coefficients (since $\ell$ is known to be an integer) we infer that, up to $n$-independent rescalings, for a given solution all the coefficients $c_{n}$ are integer multiples of the same basic quantity, $\gamma$. Dividing the recurrence relation by $\gamma$, we have $0=\nu c_{n-1} / \gamma \bmod 2$ for all $n$, because only a single term in the coefficients of (A21) is not guaranteed to be even. As an overall factor of two could be absorbed into the definition of $\gamma$ (and therefore $c_{n-1} / \gamma$ may well be odd), we conclude that $\nu=2 N$, giving

$$
\delta=\frac{2 m \alpha}{\hbar^{2} N}
$$

and

$$
\lambda=-\frac{m \alpha}{2 \hbar^{2} N}
$$

which is the known energy spectrum of hydrogen.
It is instructive to look at the detailed recurrence for the case of $\ell=0$, which includes the ground state, such that $s=2$. For $n=0$ in (A21), we obtain $c_{-1}=0$. Choosing $n=1$ in (A21) then shows that $c_{0}=0$. For $n=2$, we obtain a nontrivial relation that determines $c_{2}$ in terms of a free $c_{1}$ :

$$
c_{2}=3(1-\nu / 2) c_{1}
$$

For $\nu=2$, the smallest allowed value is $c_{2}=0$, which then implies $c_{3}=0$ at $n=3$. With two successive vanishing $c_{n}$, all the following $c_{n}$ are zero. Since $c_{1}$ may be nonzero, there is a nonzero solution, as required for a nonzero expectation value of the positive operator $\hat{r}^{2} e^{-k \hat{r}}$. A nonzero $c_{1}$ implies through (A22) that $A_{3}$ is the only nonzero coefficient, such that

$$
L_{2}\left(k, \lambda_{0}\right) \propto\left(k+\frac{2 m \alpha}{\hbar^{2}}\right)^{-3}
$$

using (A18). According to its definition (A10) as an expectation value, $L_{2}\left(k, \lambda_{0}\right)=\kappa_{0}\left(k, \lambda_{0}\right)=\omega_{0}\left(e^{-k \hat{r}}\right)$ should be the ground-state expectation value of $e^{-k \hat{r}}$, which can easily be confirmed to be of the form (A26) using the known ground-state wave function $\psi_{0}(r) \propto e^{-r / a}$ with the Bohr radius $a=\hbar^{2} /(m \alpha)$.

\section{APPENDIX B: GENERALIZATION TO HYDROGEN WITH A MAGNETIC NUCLEAR CHARGE}

Since most of the identities used in our new derivation of Kramer's relation hold true in the nonassociative case with a pointlike magnetic monopole at the center, we can easily generalize this relation. We only have to adjust the spectrum of $\hat{K}$ using (84) in (A6) and obtain

$$
\begin{aligned}
0= & \hbar^{2}(s+1)\left(\frac{s(s+2)}{4}-\ell(\ell+1)+e^{2} g^{2} / \hbar^{2}\right) \omega\left(\hat{r}^{s-1}\right) \\
& +(2 s+3) m \alpha \omega\left(\hat{r}^{s}\right)+2(s+2) m \lambda \omega\left(\hat{r}^{s+1}\right)
\end{aligned}
$$

as a generalization of (A7).

This equation takes the form

$$
\begin{aligned}
0= & \frac{\hbar^{2}}{4} \omega\left((\hat{r} f(\hat{r}))^{\prime \prime \prime}\right)-\hbar^{2}\left(\ell(\ell+1)-e^{2} g^{2}\right) \omega\left(\hat{r}^{-1} f^{\prime}(\hat{r})\right) \\
& +m \alpha \omega\left(2 f^{\prime}(\hat{r})+\hat{r}^{-1} f(\hat{r})\right)+2 m \lambda \omega\left((\hat{r} f(\hat{r}))^{\prime}\right)
\end{aligned}
$$

as a differential equation replacing (B2), which in turn implies the equation 


$$
\begin{aligned}
0= & \left(\hbar^{2} s\left(s^{2}-1-4\left(\ell(\ell+1)-e^{2} g^{2} / \hbar^{2}\right)\right)\right. \\
& -\left(4 m \alpha(2 s+1)+k\left(4 \ell(\ell+1)-4 e^{2} g^{2} / \hbar^{2}-3 s(s+1)\right)\right) \partial_{k} \\
& \left.+\left(8 m(s+1)-8 k m \alpha+3 k^{2}(1+s) \hbar^{2}\right) \partial_{k}^{2}+k\left(8 m \lambda+k^{2}\right) \hbar^{2} \partial_{k}^{3}\right) L_{s}(k, \lambda)
\end{aligned}
$$

instead of (A11).

The recurrence relation (A13) still holds with the same $C_{3}$ and $C_{2}$, while $C_{1}$ and $C_{0}$ are replaced by

$$
\begin{aligned}
C_{1}^{\prime}= & (b+n-1)\left(3 d \hbar^{2}(b+n)(b+n+1)+d \hbar^{2}\left(-4 \ell(1+\ell)+-4 e^{2} g^{2} / \hbar^{2}+3 s(1+s)\right)\right. \\
& \left.+(b+n)\left(-6 d \hbar^{2}(1+s)-8 m \alpha\right)+4 m \alpha(1+2 s)\right) \\
C_{0}^{\prime}= & -h^{2}(b+n-s)\left((b+n-s)^{2}-(2 \ell+1)^{2}+4 e^{2} g^{2} / \hbar^{2}\right) .
\end{aligned}
$$

The same choice $d=\sqrt{-8 m \lambda} / \hbar$ as in the derivation of (A19) can be used to reduce the equation to second order, and it has the same large- $n$ behavior as before. The sequence of $a_{n}$ therefore still has only finitely many nonzero elements, which is again the case if $b-s$ is an integer because the coefficient $b+n-s$ in the last term of the recurrence relation has not changed. However, there is now a second possibility if $b$ and $s$ are such that $(b+n-s)^{2}=(2 \ell+1)^{2}-4 e^{2} g^{2} / \hbar^{2}$ for some integer $n$. This condition can provide new solutions and a more complicated spectrum.

The last coefficient, $(b+n-s)^{2}-(2 \ell+1)^{2}+4 e^{2} g^{2} / \hbar^{2}$, no longer factorizes. Setting $b=0$ as before, we therefore obtain a relation,

$$
\begin{aligned}
0= & 2 d(n-2)(n-1)\left(-4 m \alpha+d(-1+n-s) \hbar^{2}\right) a_{n-2} \\
& +(n-1)\left(-4 m \alpha(2 s+1)-3 d n(1+n) \hbar^{2}+d\left(4 \ell(\ell+1)-4 e^{2} g^{2} / \hbar^{2}-3 s(1+s)\right) \hbar^{2}+n\left(8 m \alpha+6 d(1+s) \hbar^{2}\right)\right) a_{n-1} \\
& +(n-s)\left((n-s)^{2}-(2 \ell+1)^{2}+4 e^{2} g^{2} / \hbar^{2}\right) \hbar^{2} a_{n},
\end{aligned}
$$

in which the coefficient $n-1$ does not cancel out as before (for $s=2 \ell+2$ ) because the last coefficient no longer factorizes in the same way. In the previous section we have already indicated several steps in the derivation of the standard hydrogen spectrum that would no longer hold if $\ell$ [or the effective $\tilde{\ell}$ in (86) if $g \neq 0$ ] is not an integer.

More specifically, we again now look at the case of $\ell=0$ or $s=2$, comparing with the discussion at the end of the preceding section. Now, choosing $n=1$ implies a nontrivial condition, given by $a_{1}=0$, because we are no longer able to factor out $n-1$. With this value, $n=2$ is then identically satisfied. At this stage, we have the same behavior as before, with a single coefficient $\left(a_{1}\right.$ here corresponding to $c_{-1}$ before) required to be zero. At $n=3$, we obtain a linear relationship between $a_{2}$ and $a_{3}$, specifically

$$
2\left(m \alpha-d e^{2} g^{2}\right) a_{2}=e^{2} g^{2} a_{3} .
$$

The previous equation, $c_{0}=0$, would correspond to $a_{2}=0$, which is implied only if $g=0$, while $a_{3}=0$ may be implied for suitable quantized charges such that $e^{2} g^{2}$ is an integer, given the value of $d$. For generic magnetic charges $g$, and in particular for small ones such that $0 \neq e^{2} g^{2} / \hbar^{2} \ll 1, a_{2}$ and $a_{3}$ are not independent. It is then impossible to make the recurrence end with a nonzero expectation value of $e^{-k \hat{r}}$, which is a contradiction. As in the main text, we see that the quantum number $\ell=0$ is ruled out for weak magnetic charges.
[1] P. A. M. Dirac, Quantised singularities in the electromagnetic field, Proc. R. Soc. A 133, 1 (1931).

[2] M. Günaydin and B. Zumino, Magnetic charge and nonassociative algebras, in Symposium on Old and New Problems in Fundamental Physics, held in Honor of G. C. Wick (Publications of the Scuola Normale Superiore, Pisa, Italy, 1986), pp. 43-53.
[3] R. Jackiw, Three-Cocycle in Mathematics and Physics, Phys. Rev. Lett. 54, 159 (1985).

[4] R. Jackiw, Dirac's magnetic monopoles (Again), Int. J. Mod. Phys. A 19, 137 (2004).

[5] M. Günaydin, C. Piron, and H. Ruegg, Moufang plane and octonionic quantum mechanics, Commun. Math. Phys. 61, 69 (1978). 
[6] R. Blumenhagen, A. Deser, D. Lüst, E. Plauschinn, and F. Rennecke, Non-geometric fluxes, asymmetric strings and nonassociative geometry, J. Phys. A 44, 385401 (2011).

[7] D. Mylonas, P. Schupp, and R. J Szabo, Membrane sigmamodels and quantization of non-geometric flux backgrounds, J. High Energy Phys. 09 (2012) 012.

[8] I. Bakas and D. Lüst, 3-Cocycles, Non-associative starproducts and the magnetic paradigm of R-flux string vacua, J. High Energy Phys. 01 (2014) 171.

[9] D. Mylonas, P. Schupp, and R. J Szabo, Non-geometric fluxes, quasi-Hopf twist deformations and nonassociative quantum mechanics, J. Math. Phys. (N.Y.) 55, 122301 (2014).

[10] D. Mylonas, P. Schupp, and R. J Szabo, Nonassociative geometry and twist deformations in non-geometric string theory, arXiv:1402.7306.

[11] M. Bojowald, S. Brahma, and U. Büyükçam, Testing Nonassociative Quantum Mechanics, Phys. Rev. Lett. 115, 220402 (2015).

[12] M. Bojowald, S. Brahma, U. Büyükçam, J. Guglielmon, and M. van Kuppeveld, Small Magnetic Charges and Monopoles in Nonassociative Quantum Mechanics, Phys. Rev. Lett. 121, 201602 (2018).

[13] R. Haag, Local Quantum Physics (Springer-Verlag, Berlin, Heidelberg, New York, 1992).

[14] F. Bayen, M. Flato, C. Fronsdal, A. Lichnerowicz, and D. Sternheimer, Deformation theory and quantization. II. Physical applications, Ann. Phys. (N.Y.) 111, 111 (1978).

[15] C. Fronsdal, Invariant $*$-product quantization of the onedimensional Kepler problem, J. Math. Phys. (N.Y.) 20, 2226 (1979).
[16] J. M. Gracia-Bondía, Hydrogen atom in the phase-space formulation of quantum mechanics, Phys. Rev. A 30, 691 (1984).

[17] M. Bojowald, S. Brahma, U. Büyükçam, and T. Strobl, States in non-associative quantum mechanics: Uncertainty relations and semiclassical evolution, J. High Energy Phys. 03 (2015) 093.

[18] M. Bojowald, J. Guglielmon, and M. van Kuppeveld, Moments and saturation properties of eigenstates, Phys. Rev. D 103, 126005 (2021).

[19] M. Fierz, Zur Theorie magnetisch geladener Teilchen, Helv. Phys. Acta 17, 27 (1944).

[20] H. J. Lipkin, W. I. Weisberger, and M. Peshkin, Magnetic charge quantization and angular momentum, Ann. Phys. (N.Y.) 53, 203 (1969).

[21] C. J. Eliezer and S. K. Roy, The effect of a magnetic pole on the energy levels of a hydrogen-like atom, Proc. Cambridge Philos. Soc. 58, 401 (1962).

[22] S. L. Campbell et al., A Fermi-degenerate three-dimensional optical lattice clock, Science 358, 90 (2017).

[23] L. L. Vant-Hull, Experimental upper limit on the magnetic monopole moment of electrons, protons, and neutrons, utilizing a superconducting quantum interferometer, Phys. Rev. 173, 1412 (1968).

[24] R. F. Palmer and J. G. Taylor, Magnetic charge of the proton and neutron, Nature (London) 219, 1033 (1968).

[25] K. P. Jungmann, Precision muonium spectroscopy, J. Phys. Soc. Jpn. 85, 091004 (2016).

[26] M. Ahmadi et al., Observation of the hyperfine spectrum of antihydrogen, Nature (London) 548, 66 (2017).

[27] M. Ahmadi et al., Characterization of the 1S-2S transition in antihydrogen, Nature (London) 557, 71 (2018). 